\title{
Índices Parasitários de Brevimulticaecum sp. (Nematoda: Heterocheilidae) Em Potamotrygon motoro (Chondrichthyes: Potamotrygonidae) Capturados no Arquipélago do Bailique, Macapá \\ Parasitic indices of Brevimulticaecum sp. (Nematoda: Heterocheilidae) In Potamotrygon motoro (Chondrichthyes: Potamotrygonidae) Captured in the Archipelago of Bailique, Macapá
}

\author{
Antonio Carlos Freitas Souza*a; Cecile de Souza Gama ${ }^{\text {b }}$ Jociel Ferreira Costa ${ }^{a}$; Anderson Luiz Pena da Costa \\ Diego Carvalho Viana ${ }^{a}$ \\ ${ }^{a}$ Universidade Estadual do Maranhão, Programa de Pós-Graduação Stricto Sensu em Ciência Animal. MA, Brasil. \\ 'Instituto de Pesquisas Científicas e Tecnológicas do Estado do Amapá. AP, Brasil. \\ 'Instituto Macapaense de Ensino Superior. AP, Brasil. \\ *E-mail: jr_bio2005@yahoo.com.br
}

\begin{abstract}
Resumo
O número de nematódeos descritos em elasmobrânquios é relativamente pequeno, sendo encontrados majoritariamente no trato digestório. Apesar de alguns relatos descritos, a literatura a respeito ainda é considerada escassa principalmente sobre a associação parasita-hospedeiro. Diante disso, o objetivo deste trabalho foi avaliar os índices parasitários de larvas Brevimulticaecum sp. na válvula espiral de Potamotrygon motoro provenientes do arquipélago do Bailique, em Macapá no estado do Amapá, Brasil. Registrou-se em 20,41\% das raias coletadas a presença de larvas de Brevimulticaecum sp., sendo a intensidade média de 20,10 larvas/peixe e a abundância média de 4,10. São poucos os registros deste parasito em peixes no Brasil, sendo que já foi relatada sua ocorrência no estômago de P. motoro e Potamotrygon falkneri. As diferenças entre os parâmetros parasitários podem ser um indicador de alimentação e/ou diferenças comportamentais entre os sexos. Este é o primeiro estudo sobre nematóides de potamotrigonídeos na região Norte, ampliando o conhecimento da biodiversidade desta região.
\end{abstract}

Palavras-chave: Helmintos. Nematoda; Elasmobrânquio. Válvula Espiral.

\begin{abstract}
The number of nematodes described in elasmobranchs is relatively small, being found mostly in the digestive tract. Despite some reports described, the literature on this subject is still considered scarce, mainly on the parasite-host association. Therefore, the objective of this work was to evaluate the parasitic indices of Brevimulticaecum sp. the Potamotrygon motoro spiral valve from the Bailique archipelago, in Macapá in the state of Amapá, Brazil. Larvae of Brevimulticaecum sp. Were recorded in $20.41 \%$ of the rays collected, with an average intensity of 20.10 larvae / fish and an average abundance of 4.10. There are few records of this parasite in fish in Brazil, and its occurrence in the stomach of P. motoro and Potamotrygon falkneri has been reported. Differences between parasitic parameters can be an indicator of diet and / or behavioral differences between genders. This is the first study on potamotrigonid nematodes in the North, expanding knowledge of this region's biodiversity.
\end{abstract}

Keywords: Helminths. Nematoda. Elasmobranchs. Spiral Valve.

\section{Introdução}

Os elasmobrânquios de água doce da família Potamotrygonidae Garman (1913) são encontrados na maioria dos sistemas fluviais da América do Sul (CARVALHO et al., 2004). Esta família possui aproximadamente 42 espécies válidas distribuídas em quatro gêneros (SILVA; LOBODA, 2019): Paratrygon (DUMÉRIL, 1865), Plesiotrygon (ROSA, CASTELLO; THORSON, 1987), Potamotrygon (GARMAN, 1877) e Heliotrygon (CARVALHO; LOVEJOY, 2011).

Os potamotrigonídeos são monofiléticos, compartilhando especializações morfológicas e fisiológicas, tais como cintura pélvica com processo pré-pélvico, sangue com baixa concentração de uréia e diminuição da glândula retal (CARVALHO et al., 2003). São raias de médio a grande porte, com adultos possuindo variação de largura do disco em torno de 25 a $100 \mathrm{~cm}$. O gênero Potamotrygon normalmente possui cauda curta e grossa, geralmente menor que o comprimento do disco (LOBODA, 2010).

Quanto aos índices parasitários em raias da família Potamotrygonidae, os dados disponíveis ainda podem ser considerados como insuficientes quando comparados com os teleósteos. A maioria dos estudos de helmintos em Chondrichthyes é restrita a taxonomia parasitária (MENORET; IVANOV, 2009; DOMINGUES; MARQUES, 2010), evolução e filogenia (BROOKS, 1992) e descrição de ocorrência (LACERDA; TAKEMOTO; PAVANELLI, 2008).

No meio ambiente, estes parasitos normalmente não mantêm uma relação que ocasione danos irreversíveis aos hospedeiros (TAKEMOTO et al., 2004), mas de acordo com o nível de infecção parasitária, patogenicidade e dos mecanismos de compensação inerentes, esses helmintos podem ocasionar lesões mais sérias, fazendo com que possam ser utilizados como indicadores de saúde em peixes (LIZAMA et al., 2006; LACERDA;TAKEMOTO; PAVANELLI, 2009).

É importante citar que os Nematoda são heteroxênicos, e durante o seu ciclo de vida passam por vários hospedeiros intermediários, geralmente um microcrustáceo copépodo e diversos vertebrados, dentre eles, os peixes (SILVA-JÚNIOR et al., 2011). Independente do ambiente, dulcícola ou marinho, os grupos de parasitos mais comumente encontrados 
são respectivamente os Digenea, Nematoda, Monogenea e Cestoda (LUQUE; POULIN, 2007). Os níveis de parasitismo vão se diferenciar de acordo com a espécie, período sazonal e habitat (TAKEMOTO; LIZAMA, 2010).

O gênero Brevimulticaecum sp. foi relatado pela primeira vez parasitando a espécie Potamotrygon motoro (Müller \& Henle, 1841) no trabalho realizado por Rego (1979) em raias provenientes do rio Salobra, Mato Grosso (bacia do alto Paraguai, Brasil). O objetivo deste trabalho foi avaliar os índices parasitários de Brevimulticaecum sp. na válvula espiral de $P$. motoro provenientes do arquipélago do Bailique, Macapá-Amapá, Brasil.

\section{Material e Métodos}

A ilha do Parazinho (0052'41.14" N, 4959'28.22”W) é localizada na porção norte da foz do rio Amazonas, pertencente ao Arquipélago do Bailique, sendo considerado um distrito do município de Macapá, estado do Amapá, Brasil. A ilha com aproximadamente 112 hectares (Figura 1) que é alterada continuamente pela sedimentação do rio Amazonas (SOUZA-FILHO, 2005), foi declarada unidade de conservação de proteção integral, categoria Reserva Biológica em 21 de janeiro de 1985.

Figura 1 - Mapa com a localização dos pontos de coletas de amostras na reserva biológica do Parazinho, Amapá, Brasil
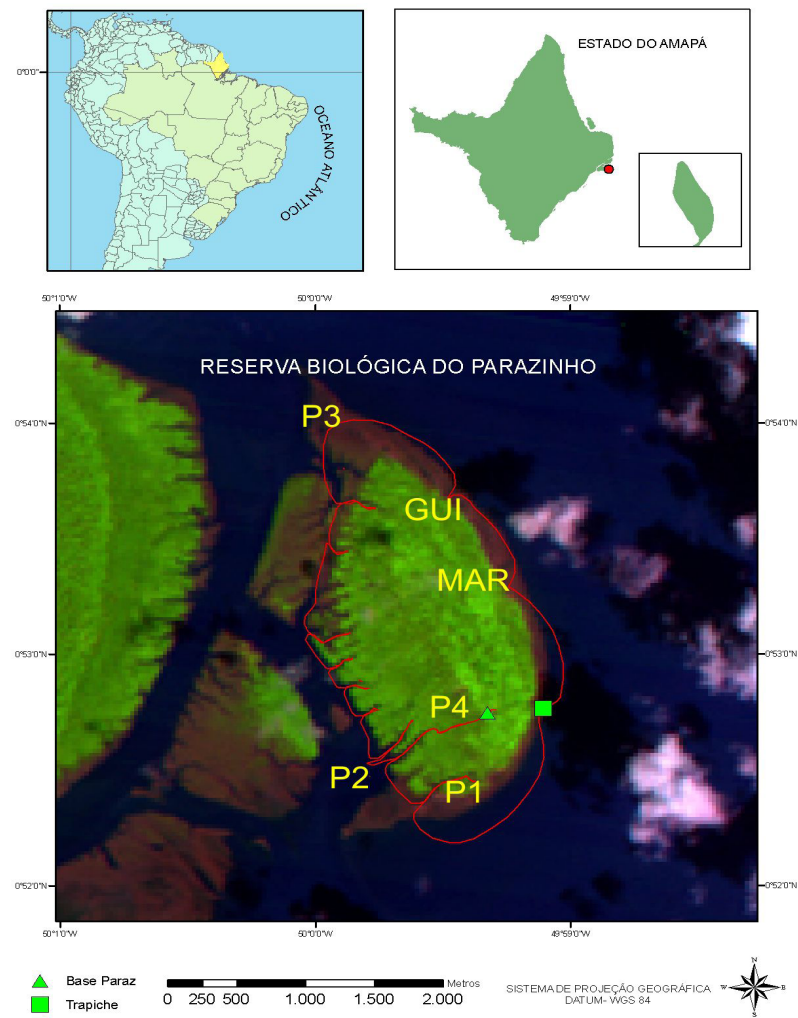

Fonte: Os autores.

Este trabalho faz parte de um estudo realizado a partir do Termo de Cooperação Técnica Nº05/2010-SEMA/IEPA com intuito de adequar a gestão da REBIO Parazinho e construção do seu Plano de Manejo. Foram realizadas seis coletas durante os meses de março de 2012 a janeiro de 2013, onde foram escolhidos seis pontos de amostras em todo o perímetro da ilha. Foram instalados espinhéis de fundo, vistoriados a cada maré vazante, além da utilização de tarrafas, zagaias e tapagem de canais de maré para a coleta.

As raias capturadas foram anestesiadas com Eugenol, realizada eutanásia e dissecadas para a retirada de seu estômago. Os estômagos foram fixados em formalina $10 \%$ neutralizada com carbonato de cálcio. Os exemplares de raias foram fixados em formalina $10 \%$. Após 7 dias as raias eram lavadas com água corrente e transferidas para solução de álcool 70\%.

Os espécimes de raias capturados foram identificados através de seu padrão de coloração dorsal, por meio de comparações com espécimes depositados na Coleção Científica Fauna do Amapá, pertencente ao Instituto de Pesquisas Científicas e Tecnológicas do Estado do Amapá (IEPA), descrições originais das espécies e, quando necessário foi realizado contato com especialistas para auxílio.

Os órgãos coletados foram colocados separadamente em placas de Petri contendo soro fisiológico, para análise individualizada em estéreo microscópio (Medilux SQZ-DS4). Os procedimentos de coleta, fixação e quantificação dos parasitos seguiram as recomendações de Eiras et al. (2006) e Thatcher (2006). Os nematoides coletados foram limpos, com o auxílio de pincel e bisturi, sendo contabilizados e logo em seguida fixados com AFA (2\% de ácido acético glacial, 3\% de formol a $37 \%$ e $95 \%$ de álcool etílico a $70 \%$ ), aquecido a aproximadamente $65^{\circ} \mathrm{C}$, sendo guardados em tubos de ensaio com tampa durante o período de 48 horas, onde foram transferidos para outro recipiente contendo Álcool 70\% com o intuito de conservá-los para posterior análise e identificação.

O exame morfológico seguiu os padrões de rotina da ictioparasitologia, sendo os vermes desidratados com bateria crescente de álcool $(70 \%, 80 \%, 90 \%$ e $100 \%)$, clarificados com Lactofenol e observados em Microscopia óptica, a identificação dos parasitos foi baseada nos trabalhos de Marques et al. (2003), Marques e Brooks (2003), Thatcher (2006).

A prevalência, a abundância parasitária e a intensidade média de infecção foram calculadas de acordo com Bush et al. (1997). Os cálculos foram realizados com o auxílio do programa BioEstat 5.0.

\section{Resultados e Discussão}

Foram examinados 49 espécimes de P. motoro, sendo 18 fêmeas e 31 machos, que apresentaram intervalo de comprimento total variando entre $44,4 \mathrm{~mm}$ a $551 \mathrm{~mm}$ e 29 mm a $699 \mathrm{~mm}$ respectivamente. Quanto ao peso, as fêmeas tiveram variação entre $760 \mathrm{~g}$ e $4000 \mathrm{~g}$, e os machos entre 290 g a 3880 g. Do total de espécimes analisados, 10 estavam parasitados, o que representa uma prevalência 20,41\%. Se separados por sexo, $22,22 \%$ das fêmeas e $19,35 \%$ dos machos foram positivos para o parasitismo. Esta diferença foi testada através do $X^{2}$ resultando em não significativo $\left(X^{2}=1,22\right)$. 
Quando avaliada a variação em relação ao período chuvoso e a estiagem amazônica, foi possível encontrar prevalência de $29,17 \%$ e $12 \%$ respectivamente. Esta diferença foi testada através do $\mathrm{X}^{2}$ resultando em significativo $\left(\mathrm{X}^{2}=0,024\right)$.

Nos indivíduos parasitados $(\mathrm{n}=10)$ foram quantificados um total de 201 parasitos (Quadro 1), com intensidade média de 20,1 larvas/peixe, e valores mínimos e máximos variando entre 1 a 143 parasitos. Quando separados por sexo, as fêmeas tiveram intensidade média de 47,5 larvas/peixe e abundância de 10,56, e os machos tiveram intensidade média de 1,83 larvas/peixe e abundância de 0,35 . Os parasitos encontrados estavam todos em estágio larval e pertenciam ao gênero Brevimulticaecum sp.

Quadro 1 - Comprimento total (Lt), Comprimento do disco (Ld), peso e número de parasitos encontrados em machos e fêmeas da espécie P. motoro proveniente da ilha do Parazinho, Amapá, Brasil

\begin{tabular}{|c|c|c|c|c|c|}
\hline & & Lt (mm) & Ld (mm) & Peso (g) & $\begin{array}{l}\text { Número } \\
\text { Parasitos }\end{array}$ \\
\hline \multirow{18}{*}{ 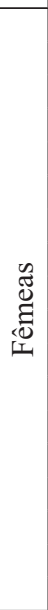 } & 1 & 551 & 312 & 1500 & 143 \\
\hline & 2 & 79,2 & 43,4 & 4000 & 0 \\
\hline & 3 & 74,3 & 51,4 & 3750 & 0 \\
\hline & 4 & 46,6 & 25,5 & 1000 & 0 \\
\hline & 5 & 70,4 & 38,7 & 3000 & 0 \\
\hline & 6 & 48,9 & 27,2 & 1020 & 0 \\
\hline & 7 & 64,8 & 35,2 & 2320 & 0 \\
\hline & 8 & 58,9 & 32,9 & 1650 & 0 \\
\hline & 9 & 51,1 & 27,8 & 1340 & 0 \\
\hline & 10 & 65 & 35,8 & 2190 & 0 \\
\hline & 11 & 74 & 43,8 & 3410 & 0 \\
\hline & 12 & 44,4 & 25,8 & 760 & 2 \\
\hline & 13 & 65,2 & 38,7 & 3140 & 0 \\
\hline & 14 & 65,2 & 38,7 & 3140 & 1 \\
\hline & 15 & 74 & 43,8 & 3380 & 0 \\
\hline & 16 & 51,7 & 31 & 1390 & 44 \\
\hline & 17 & 49,8 & 27,4 & 1010 & 0 \\
\hline & 18 & 49,9 & 27,6 & 1020 & 0 \\
\hline \multirow{31}{*}{$\begin{array}{l}\stackrel{0}{0} \\
\frac{d}{0} \\
\stackrel{\Xi}{2}\end{array}$} & 1 & 687 & 395 & 3,000 & 0 \\
\hline & 2 & 699 & 369 & 2,500 & 0 \\
\hline & 3 & 715 & 365 & 2,000 & 0 \\
\hline & 4 & 43,6 & 24,3 & 750 & 0 \\
\hline & 5 & 68,5 & 38,8 & 2650 & 0 \\
\hline & 6 & 56,8 & 33,8 & 1750 & 0 \\
\hline & 7 & 45,3 & 25 & 840 & 0 \\
\hline & 8 & 51,7 & 28,1 & 1050 & 0 \\
\hline & 9 & 46,2 & 24,9 & 700 & 0 \\
\hline & 10 & 41,2 & 24,4 & 600 & 0 \\
\hline & 11 & 63,9 & 37,1 & 2260 & 0 \\
\hline & 12 & 48,5 & 27,1 & 860 & 0 \\
\hline & 13 & 54,2 & 31,7 & 1520 & 0 \\
\hline & 14 & 43,7 & 24,6 & 790 & 0 \\
\hline & 15 & 48,8 & 35,4 & 1780 & 0 \\
\hline & 16 & 45,5 & 28,1 & 1030 & 0 \\
\hline & 17 & 48,8 & 27,1 & 940 & 0 \\
\hline & 18 & 55,6 & 32,7 & 1490 & 0 \\
\hline & 19 & 52,6 & 29,4 & 1140 & 0 \\
\hline & 20 & 45,2 & 23,2 & 670 & 0 \\
\hline & 21 & 29 & 22,5 & 600 & 0 \\
\hline & 22 & 58,3 & 32,8 & 1690 & 0 \\
\hline & 23 & 53,4 & 29,5 & 1120 & 0 \\
\hline & 24 & 54,3 & 31,4 & 1620 & 0 \\
\hline & 25 & 48 & 27,2 & 1030 & 2 \\
\hline & 26 & 54,2 & 30,8 & 1420 & 2 \\
\hline & 27 & 65,6 & 36,5 & 2290 & 2 \\
\hline & 28 & 50,5 & 28,8 & 1060 & 2 \\
\hline & 29 & 77,5 & 44,8 & 3880 & 1 \\
\hline & 30 & 33 & 18,4 & 290 & 0 \\
\hline & 31 & 46,5 & 24,8 & 750 & 2 \\
\hline
\end{tabular}

Fonte: Dados da pesquisa.
Larvas do gênero Brevimulticaecum sp. já foram relatadas para as espécies de potamotrigonídeos, Lacerda, Takemoto e Pavanelli (2009) relataram sua presença no estômago de Potamotrygon falkneri provenientes do rio Paraná. Lemos (2011) também encontrou em Potamotrygon cf. histrix no mesmo rio. No Checklist proposto por Gama (2016) larvas de Brevimulticaecum sp. são citadas em P. motoro e Potamotrygon cf. castexi ambos do rio Manu, Peru. Apesar destes achados, não há relatos na literatura para raias provenientes do norte do Brasil, sendo este o primeiro trabalho.

A presença destes Nematoda em raias pode ser justificada principalmente pelo seu hábito alimentar, que apesar de ser generalista, tem preferência por pequenos crustáceos (SHIBUYA et al., 2009) que são hospedeiros intermediários dos nematóides (ANDERSON, 2000). Gama e Rosa (2015) comprovaram que a dieta de $P$. motoro proveniente do arquipélago do Bailique, consiste, prioritariamente, em camarões e caranguejos além de pequenos teleósteos, justificando os achados no presente estudo.

A localização geográfica pode ser considerada outro fator importante, pois os períodos sazonais da Amazônia, que são caracterizados pela estiagem (águas baixas) e a cheia (águas altas) podem ter influenciado os índices parasitários, principalmente quando relacionado à disponibilidade de alimentos.

Nos períodos de seca, existe a tendência de agregação dos hospedeiros intermediários, tornando-se um recurso pouco variado e limitado localmente, o que pode levar a competição intra e interespecífica, tornando as dietas mais específicas (GAMA; ROSA, 2015). Por outro lado, no período chuvoso, encontra-se uma grande variedade de recursos alimentares em todos os locais, o que pode vir a justificar índices parasitários superiores neste período na área de estudo. Corroborando estas informações, Vital et al. (2011) encontraram índices parasitários superiores nas estações de enchente e cheia para Procamallanus inopinatus em Pygocentrus nattereri provenientes do lago Piranha (AM), justificando que nesta estação ocorre a melhora das condições ambientais na Amazônia para vários organismos aquáticos. Dessa forma, existe uma grande quantidade de indivíduos, o que viabiliza o encontro das formas larvais infectantes com seus hospedeiros, permitindo assim que o ciclo de vida das diversas espécies se complete.

Quanto à patogenicidade, normalmente peixes alojam parasitos não letais, sendo sua abundância normalmente controlada pelo sistema imunológico (TAKEMOTO et al., 2004). Porém a dinâmica dos ecossistemas aquáticos, associada à degradação ambiental, configura um fator estressante ao meio biótico, que vem interferir na vida dos peixes e parasitos (PANTOJA; FLORES; TAVARES-DIAS, 2015). Apesar da baixa amostragem, a dinâmica parasitária já demonstrou diferenças entre os sexos no presente estudo. Essa diferença entre intensidade média de infecção entre fêmeas e machos encontrada já foi relatada por Lizama et al. 
(2005). Ainda é importante citar que essas distinções podem ocorrer a partir da interação de diversos fatores, tais como a disponibilidade de alimento, a espécie hospedeira, fatores imunológicos, competição intra e interespecífica, mudanças na qualidade físico-química da água, entre outros (TAKEMOTO; LIZAMA, 2010).

\section{Conclusão}

As diferenças entre os parâmetros parasitários podem ser um indicador de alimentação e/ou diferenças comportamentais entre os sexos. Até o presente momento nenhum trabalho havia sido realizado sobre a fauna parasitária de potamotrigonídeos na área de estudo, contribuindo para o conhecimento da biodiversidade.

\section{Referências}

ANDERSON, R.C. Nematode parasites of vertebrates: their development and transmission. Wallingford: CABI Publishing, 2000.

BROOKS, D.R. Origins, diversification, and historical structure of the helminth fauna inhabiting neotropical freshwater stingrays (Potamotrygonidae). J. Parasitology., v.78, n.4, p.588-595, 1992. doi: $10.2307 / 3283533$

BUSH, A.O. et al. Parasitology meets ecology on its own terms. J. Parasitology., v.83, p.575-583, 1997.

CARVALHO, M.R.; LOVEJOY, N.R.; ROSA, R.S. Family Potamotrygonidae.. In: REIS, R.E.; FERRARIS JR, C.J.; KULLANDER, S.O. Checklist of the freshwater fishes of south and Central America. Porto Alegre: EDIPUCRS, 2003. p.22-29

CARVALHO, M.R.; MAISEY, J.G.; GRANDE, L. Freshwater stingrays of the Green River Formation of Wyoming (Early Eocene), with the description of a new genus and species and ananalysis of its phylogenetic relationships (Chondrichthyes: Myliobatiformes). Bull. Am. Mus. Nat. Hist., v.284, p.136, 2004.

DOMINGUES, M.V. ; MARQUES, F.P.L. Ergasilus trygonophilus sp. nov. (Copepoda: Ergasilidae) a branchial parasite of freshwater stingrays (Potamotrygonidae) from state of Pará, Brazil. Soc. Bras. Zool., v.27, n.5, p.829-833, 2010. doi: 10.1590/S1984-46702010000500020

EIRAS, J.C.; TAKEMOTO, R.M.; PAVANELLI, G.C. Métodos de estudo e técnicas laboratoriais em parasitologia de peixes. Maringá: EDUEM, 2006.

GAMA, C.S.; ROSA, R.S. Uso de recursos e dieta das raias de água doce (Chondrichthyes, Potamotrygonidae) da Reserva Biológica do Parazinho, AP. Biota Amazônia, v.5, n.1, p.90-98, 2015. doi: 10.18561/2179-5746/biotaamazonia.v5n1p90-98

GAMA, C.S. Parasitas de raias de água doce (Potamotrygonidae): estado atual do conhecimento e lista de espécies. In: LASSO, C.A. et al. Rayas de água Dulce (Potamotrygonidae) de Suramérica. Parte II: Colombia, Brasil, Perú, Bolivia, Paraguay, Uruguay y Argentina. Bogotá: Instituto de Investigación de Recursos Biológicos Alexander von Humboldt (IAvH), 2016. p.67-82

LACERDA, A.C.F.; TAKEMOTO, R.M.; PAVANELLI, G.C. Digenea, Nematoda, Cestoda and Acanthocephala, parasites in Potamotrygonidae (Chondrichthyes) from the upper Paraná River flood plain, states of Paraná and Mato Grosso do Sul, Brazil. CheckList, v.4, n.2, p.115-122, 2008.

LACERDA, A.C.F.; TAKEMOTO, R.M.; PAVANELLI, G.C. Ecology of endoparasites of the fluvial stingray Potamotrygon falkneri (Chondrichthyes: Potamotrygonidae) from the upper Paraná River flood plain, Brazil. J. Biology, v.69, n.2, p.297-303, 2009. doi: 10.1590/S1519-69842009000200009

LEMOS, J.R.G. Índices parasitários e parâmetros fisiológicos de arraias Cururu (Potamotrygon cf. histrix) exportadas como peixes ornamentais: ferramentas para avaliação do estado de saúde da espécie. Manaus: UFAM, 2011.

LIZAMA, M.A.; TAKEMOTO, R.M.; PAVANELLI, G.C. Influence of host sex and age on infracommunities of metazoan parasites of Prochilodus lineatus (Valenciennes, 1836) (Prochilodontidae) of the Upper Paraná River floodplain, Brazil. Parasite, v.12, n.4, p.299-304, 2005. doi: 10.1051/ parasite/2005124299

LIZAMA, M.A.; TAKEMOTO, R.M.; PAVANELLI, G.C. Parasitism influence on the hepato, splenosomatic and weight/ length relation and relative condition factor of Prochilodus lineatus (Valenciennes, 1836) (Prochilodontidae) of the Upper Paraná River floodplain, Brazil. Rev. Bras. Parasitol. Vet., v.15, n.3, p.116-122, 2006.

LOBODA, T.S. Revisão taxonômica e morfológica de Potamotrygon motoro (Müller \& Henle, 1841) na bacia Amazônica (Chondrichthyes: Myliobatiformes: Potamotrygonidae). São Paulo: USP, 2010.

LUQUE, J.L.; POULIN, R. Metazoan parasite species richness in Neotropical fishes: hotspots and the geography of biodiversity. Parasitology, v.134, p.865-878, 2007. doi: 10.1017/ S003118200700227

MARQUES, F.P.L.; BROOKS, D.R. Taxonomic review of Rhinebothroides (Eucestoda: Tetraphyllidea: Phyllobothriidae), parasites of freshwater stingrays (Rajiformes: Myliobatoidei: Potamotrygonidae). J. Parasitol. v.89, p.944-1017, 2003.

MARQUES, F.P.L.; BROOKS, D.R.; ARAÚJO, M.L.G. Systematics and phylogeny of Potamotrygon ocestus (Platyhelminthes, Tetraphyllidea, Onchobothriidae) with descriptions of three new species from freshwater potamotrygonids (Myliobatoidea, Potamotrygonidae). Zoologica Scripta, v.32, p.367-396, 2003. doi: 10.1046/j.1463-6409.2003.00111.x

MENORET, A.; IVANOV, V.A. A new species of Tetraphyllidean (Cestoda) from the Largest port River stingray, Potamotrygon falkneri (Potamotrygonidae: Chondrichthyes), from the Paraná basin. J. Parasitol., v.95, n.4, p.994-999, 2009. doi: 10.1645/GE1850.1

PANTOJA, W.M.F.; FLORES, L.V.; TAVARES-DIAS, M. Parasites component community in wild population of Pterophyllum scalare Schultze, 1823 and Mesonauta acora Castelnau, 1855, cichlids from the Brazilian Amazon. J. Appl. Ichthyol., v.31, p.1043-1048, 2015. doi: 10.1111/jai.12903

REGO, A.A. Contribuição ao conhecimento dos helmintos de raias fluviais Paratrygonidae. Rev. Bras. Biol., v.39, n.4, p.879890, 1979.

SHIBUYA, A.; ARAÚJO, M.L.G.; ZUANON, J.A.S. Analysis of stomach contents of freshwater stingrays (Elasmobranchii, Potamotrygonidae) from the middle Negro River, Amazonas, Brazil. Pan-American J. Aquatic Scie, v.4, n.4, p.466-475, 2009.

SILVA, J.P.C.B. da; LOBODA, T.S. Potamotrygon marquesi, a new species of neotropical freshwater stingray (Potamotrygonidae) from the Brazilian Amazon Basin. J. Fish Biology, v.95, n.2, 2019. doi: $10.1111 / \mathrm{jfb} .14050$

SILVA-JÚNIOR, A.C.S.; RAMOS, J.S.; GAMA, C.S. Parasitismo de larvas de anisakidae em Acestrorhynchus lacustris da Área de Proteção Ambiental do rio Curiaú, Macapá, estado do Amapá. Rev. Bras. Eng. Pesca, v.6, n.2, p.1-10, 2011. doi: 10.18817/ 
repesca.v6i 2.300

SOUZA-FILHO, P.W.M. Costa de manguezais de macromaré da Amazônia: Cenários morfológicos, mapeamento e quantificação de áreas usando dados de sensores remotos. Rev. Bras. Geofisica, v.23, n.4, p.427-435, 2005. doi: 10.1590/S0102261X2005000400006

TAKEMOTO, R.M.; LIZAMA, M. de. Helminth fauna of fishes from the Upper Paraná river floodplain, Brazil fauna helmíntica de peces de alta planície del rio paraná, Brasil. Neotrop. Helminthol., v.4, n.1, p.5-8, 2010.
TAKEMOTO, R.M.et al. Parasitas de peixes de águas continentais. In: RANZANI-PAIVA, M.J.T.; TAKEMOTO, R.M.; LIZAMA, M.A.P. Sanidade de Organismos Aquáticos. São Paulo: Editora Varela, 179-197, 2004.

THATCHER, V.E. Amazon Fish Parasites. 2ed. Sofia-Moscow: Pensoft Publishers, 2006.

VITAL, J.F.et al. Sazonalidade da fauna de metazoários de Pygocentrus nattereri (Kner, 1858) no lago Piranha (Amazonas, Brasil) e a avaliação de seu potencial como indicadora da saúde do ambiente. Biota Neotrop., v.11, n.1, p.199-204, 2011. doi: 10.1590/S1676-06032011000100021. 